\title{
Light-year scale radio cores in four LINER galaxies
}

\author{
M. E. Filho ${ }^{1}$, P. D. Barthel ${ }^{1}$, and L. C. $\mathrm{Ho}^{2}$ \\ 1 Kapteyn Astronomical Institute, PO Box 800, 9700 AV Groningen, The Netherlands \\ 2 The Observatories of the Carnegie Institution of Washington, 813 Santa Barbara St., Pasadena CA 91101, USA
}

Received 9 November 2001 / Accepted 23 January 2002

\begin{abstract}
The LINER galaxies NGC 2911, NGC 3079, NGC 3998, and NGC 6500 were observed at 5 GHz with the European VLBI Network at a resolution of 5 milliarcsecond and found to possess flat-spectrum, variable, high-brightness temperature $\left(T_{\mathrm{B}}>10^{8} \mathrm{~K}\right)$ radio cores. These radio characteristics reinforce the view that these LINERs host central engines associated with active galactic nuclei.
\end{abstract}

Key words. galaxies: active - galaxies: nuclei - radio continuum: galaxies

\section{Introduction}

As many as $40 \%$ of all nearby galaxies display some level of nuclear activity qualitatively resembling that seen in accretion-powered active nuclei (Ho et al. 1997b; Ho 1999a). The activity manifests itself in objects such as Seyfert nuclei and low-ionization nuclear emission-line regions (LINERs; Heckman 1980). LINERs differ from Seyferts in that they display characteristically stronger low-ionization optical forbidden lines. The source of the ionization responsible for the optical emission in LINERs is still under debate. Models which invoke shock heating (Fosbury et al. 1978; Heckman 1980; Dopita \& Sutherland 1995), stellar photoionization (Terlevich \& Melnick 1985; Shields 1992; Filippenko \& Terlevich 1992; Terlevich et al. 1992; Barth \& Shields 2000), and aging starbursts (Alonso-Herrero et al. 2000) have been proposed as possible ionization mechanisms. There is, however, growing evidence that a substantial fraction of the LINER population simply constitute the local low-luminosity equivalent of "classical" active galactic nuclei (AGN) such as quasars and luminous Seyfert galaxies (see review by Ho 2001). This is borne out by the detection of weak radio cores (Heckman 1980; Sadler et al. 1989; Wrobel \& Heeschen 1991; Slee et al. 1994; Nagar et al. 2000; Falcke et al. 2000), by the nature of the ultraviolet (Maoz et al. 1995, 1998; Barth et al. 1998) and X-ray radiation (Ptak et al. 1999; Terashima et al. 2000; Halderson et al. 2001; Ho et al. 2001), and by the presence of broad $\mathrm{H} \alpha$ lines in total (Ho et al. 1997c) as well as polarized flux (Barth et al. 1999). If many LINERs are in fact true AGNs, this would have repercussions on the faint end of the AGN luminosity

Send offprint requests to: M. E. Filho, e-mail: mercedes@astro.rug.nl function, and consequently on galaxy evolution and the cosmic X-ray background.

Here we report on an investigation of the radio morphologies of four LINER galaxies on milliarcsecond (mas) scales. Observations with the European VLBI Network $(\mathrm{EVN})$ were used to obtain images of the light-year scale structure in the radio cores of these four LINERs suspected to be powered by AGNs.

This paper adopts a Hubble constant of $H_{0}=$ $75 \mathrm{~km} \mathrm{~s}^{-1} \mathrm{Mpc}^{-1}$ and defines the spectral index, $\alpha$, such that the flux density $F_{\nu} \propto \nu^{-\alpha}$.

\section{Sample selection}

The four LINER galaxies studied here (Table 1) were taken from the Palomar spectroscopic survey of bright, northern galaxies (Filippenko \& Sargent 1985; Ho et al. 1995, 1997a,b,c). They were selected as having fairly bright radio emission, with significant contributions from an unresolved component on arcsecond (VLA) scales. Optical images of NGC 2911, NGC 3079 and NGC 3998 can be seen in the Sandage \& Bedke (1994) Carnegie Atlas of Galaxies, and an image of NGC 6500 is available in González Delgado \& Pérez (1996). Various lines of evidence, to be discussed below, suggest that accretion-driven power plays a role in the nuclei of these four galaxies.

Early VLBI experiments conducted on these sources have yielded some correlated flux densities on $\mathrm{M} \lambda$ baselines (van Breugel et al. 1981; Jones et al. 1981; Hummel et al. 1982). However, images permitting assessment of morphological properties and brightness temperatures had not been obtained. We therefore conducted EVN observations to obtain such images and to quantify the contribution of the radio emission arising from the AGN and the 
Table 1. Target galaxies. Column 1: source name. Column 2: spectral class of the nucleus from Ho et al. (1997a), where $\mathrm{L}=$ LINER, $\mathrm{S}=$ Seyfert, 1.9 = weak broad $\mathrm{H} \alpha$ emission line present, and $2=$ no broad emission line; NGC 3079 falls on the borderline between Seyferts and LINERs. Columns 3 and 4: optical position from NED (NASA/IPAC Extragalactic Database). Column 5: adopted distance from Tully (1988), who also uses our value of $H_{0}$. Column 6: Hubble type from NED. Column 7: Green Bank 1.4 GHz flux density from White \& Becker (1992), 12' resolution. Column 8: NVSS 1.4 GHz flux density from Condon et al. (1998), 45" resolution. Column 9: FIRST 1.4 GHz flux density from Becker et al. (1995), 5" resolution. Column 10: Green Bank 4.9 GHz flux density from Becker et al. (1991), 3!. 5 resolution.

\begin{tabular}{|c|c|c|c|c|c|c|c|c|c|}
\hline Source & $\begin{array}{c}\text { Spectral } \\
\text { Class } \\
(2)\end{array}$ & $\begin{array}{c}\mathrm{RA}(\mathrm{J} 2000) \\
\left(\begin{array}{c}\mathrm{h} \mathrm{m} \mathrm{s} \\
\text { ( }\end{array}\right) \\
(3)\end{array}$ & $\begin{array}{c}\operatorname{Dec}(\mathrm{J} 2000) \\
\left({ }^{\circ}{ }^{\prime \prime \prime}\right) \\
(4)\end{array}$ & $\begin{array}{c}D \\
(\mathrm{Mpc}) \\
(5)\end{array}$ & $\begin{array}{l}\text { Hubble } \\
\text { Type } \\
(6)\end{array}$ & $\begin{array}{c}\text { GB } 1.4 \\
(\mathrm{mJy}) \\
(7)\end{array}$ & $\begin{array}{c}\text { NVSS } 1.4 \\
(\mathrm{mJy}) \\
(8)\end{array}$ & $\begin{array}{c}\text { FIRST } 1.4 \\
(\mathrm{mJy}) \\
(9)\end{array}$ & $\begin{array}{c}\text { GB } 4.9 \\
(\mathrm{mJy}) \\
(10)\end{array}$ \\
\hline NGC 2911 & $\overline{\mathrm{L} 2}$ & 093346.10 & 100908.5 & 42.2 & S0: pec & $\cdots$ & 58.6 & $\cdots$ & 73 \\
\hline NGC 3079 & $\mathrm{~S} 2 / \mathrm{L} 2$ & 100157.30 & 554054.0 & 20.4 & $\mathrm{SBc}$ & 845 & 770.7 & 293.0 & 321 \\
\hline NGC 3998 & L1.9 & 115756.112 & 552712.74 & 21.6 & S0? & 126 & 101.4 & 98.5 & 82 \\
\hline NGC 6500 & L2 & 175559.771 & 182018.32 & 39.7 & Sab: & 224 & 182.9 & $\ldots$ & 176 \\
\hline
\end{tabular}

Table 2. Measured radio parameters. Column 1: source name. Column 2: beam size $(F W H M)$. Column 3: position angle of the beam. Column 4: rms noise of the image. Column 5: peak $5 \mathrm{GHz}$ flux density. Column 6: integrated $5 \mathrm{GHz}$ flux density.

\begin{tabular}{|c|c|c|c|c|c|}
\hline Source & $\begin{array}{c}\text { Beam Size } \\
(\text { mas } \times \text { mas }) \\
(2)\end{array}$ & $\begin{array}{l}\mathrm{PA} \\
\left({ }^{\circ}\right) \\
(3)\end{array}$ & $\begin{array}{c}\mathrm{rms} \\
\left(\mathrm{mJy}^{\left.\text {beam }^{-1}\right)}\right. \\
(4)\end{array}$ & $\begin{array}{c}F_{\text {peak }} \\
(\mathrm{mJy}) \\
(5)\end{array}$ & $\begin{array}{c}F_{\text {int }} \\
(\mathrm{mJy}) \\
(6)\end{array}$ \\
\hline NGC 2911 & $8.4 \times 5.7$ & 50.4 & 0.19 & 18.7 & 19.9 \\
\hline NGC 3079 & $7.2 \times 5.1$ & 58.5 & 0.20 & 13.8 & 14.8 \\
\hline NGC 3998 & $7.4 \times 4.7$ & 25.9 & 0.28 & 78.2 & 83.0 \\
\hline NGC 6500 & $10.1 \times 3.6$ & 42.1 & 0.24 & 68.5 & 83.9 \\
\hline
\end{tabular}

starburst components. In addition, it was hoped that masscale structure could be traced out to large (kpc) scales. We note, in passing, that a typical EVN $5 \mathrm{GHz}$ resolving beam of 5 mas translates to $1.5-3$ light years for the sources under study.

\section{Observations and data reduction}

The observations of NGC 3998 and NGC 6500 were performed on 1997 June 5-6, while NGC 2911 and NGC 3079 were observed on 1997 June 9, all with the EVN Mark III system at $5 \mathrm{GHz}$. We combined 14 channels of $4 \mathrm{MHz}$ each to achieve a total bandwidth of $56 \mathrm{MHz}$. The requested antennas were Effelsberg, Jodrell Bank Mk2, Medicina, Noto, Torun, Westerbork, and Onsala, but unfortunately only the first five yielded useful data. Total integration time, aiming for optimal $u v$-coverage, was about 4 hours per galaxy. Cross-correlation employed the Bonn (MPIfR) Mark III correlator.

All of the Onsala data were lost, and the data from the Westerbork array were also lost due to wrong polarization observation. The loss of these two antennas was equivalent to losing $47 \%$ of our data. Furthermore, more than half of the 14 Torun channels were lost during the observations of NGC 3998 and NGC 6500. Following cross correlation, subsequent data reduction was performed at JIVE in Dwingeloo. After minor flagging, the data were calibrated, fringe fitted, and Fourier transformed using standard tools in AIPS (van Moorsel et al. 1996). Typical angular resolution achieved was 5-8 mas (Gaussian FWHM). The uncertainty of the absolute amplitude calibration, mainly due to data noise and uncertainties in the primary flux density calibrator, is estimated to be $5 \%-10 \%(1 \sigma)$.

\section{Results}

All four LINERs were detected at $5 \mathrm{GHz}$ and found to display strong $(\sim 20-80 \mathrm{mJy})$ point sources. Table 2 lists the properties of the maps and the main measured quantities, while Table 3 summarizes the derived quantities. Weak extended emission, at a few percent of the peak level, is observed in NGC 3079, NGC 3998, and NGC 6500; this will be discussed below. The deconvolved images are not shown because they suffer from sidelobe remnants and noise peaks. Because our maps are dominated by a highly compact central core, and because we are not confident about the robustness of the faint extended emission, we have chosen not to perform detailed fits to obtain deconvolved source sizes.

Peak brightness temperatures were computed as

$T_{\mathrm{B}}=\frac{F_{\text {peak }} c^{2}}{2 k_{\mathrm{B}} \nu^{2} \Omega^{2}} \quad(\mathrm{~K})$,

where $F_{\text {peak }}$ is the peak $5 \mathrm{GHz}$ flux density, $c$ is the speed of light, $k_{\mathrm{B}}$ is Boltzmann's constant, $\nu$ is the observing frequency, and $\Omega^{2}$ is the upper limit to the source size. We conservatively regard all the cores to be unresolved and give upper limits to their sizes, equivalent to half of the 
Table 3. Properties of the radio cores. Column 1: source name. Column 2: monochromatic power of the core at $5 \mathrm{GHz}$, computed from the peak flux. Column 3: spectral luminosity at $5 \mathrm{GHz}, L_{5} \mathrm{GHz} \equiv \nu P_{5} \mathrm{GHz}$. Column 4: radius. Column 5: peak brightness temperature. Column 6: variability information (see text). Column 7: non-simultaneous 2- or 3-point radio spectral index. Column 8: references used to calculate $\alpha_{\mathrm{r}}$ : (1) Slee et al. (1994) (2.3 and 8.4 GHz); (2) Trotter et al. (1998) (5, 8 and 22 GHz); (3) Wrobel \& Heeschen (1984) (1.5, 4.9 and 15 GHz); (4) Nagar et al. (2000) and this paper (5 and 15 GHz). Column 9: the logarithmic ratio of the flux densities at $60 \mu \mathrm{m}$ and $1.4 \mathrm{GHz}$. Column 10: the infrared spectral index between 25 and $60 \mu \mathrm{m}$.

\begin{tabular}{|c|c|c|c|c|c|c|c|c|c|}
\hline Source & $\begin{array}{c}P_{5 \mathrm{GHz}} \\
\left(\mathrm{W} \mathrm{Hz}^{-1}\right) \\
(2)\end{array}$ & $\begin{array}{c}L_{5 \mathrm{GHz}} \\
\left(\mathrm{erg} \mathrm{s}^{-1}\right) \\
(3)\end{array}$ & $\begin{array}{c}r \\
(\text { lty } \times \text { lty }) \\
(4)\end{array}$ & $\begin{array}{c}T_{\mathrm{B}} \\
(\mathrm{K}) \\
(5)\end{array}$ & Variable & (7) & Ref. & (9) & $(10)$ \\
\hline NGC 2911 & $3.98 \times 10^{21}$ & $1.99 \times 10^{38}$ & $<1.4 \times 0.9$ & $>1 \times 10^{8}$ & yes & 0.21 & 1 & 0.69 & $<1.11$ \\
\hline NGC 3079 & $6.87 \times 10^{20}$ & $3.44 \times 10^{37}$ & $<0.6 \times 0.4$ & $>1 \times 10^{8}$ & yes? & $-1.68 / 0.82$ & 2 & 1.81 & 3.02 \\
\hline NGC 3998 & $4.37 \times 10^{21}$ & $2.19 \times 10^{38}$ & $<0.7 \times 0.4$ & $>6 \times 10^{8}$ & yes & $0.15 / 0.32$ & 3 & 0.75 & 1.78 \\
\hline NGC 6500 & $1.29 \times 10^{22}$ & $6.45 \times 10^{38}$ & $<1.6 \times 0.6$ & $>5 \times 10^{8}$ & yes & -0.03 & 4 & 0.54 & 2.12 \\
\hline
\end{tabular}

Gaussian $F W H M$ of the synthesized beam. Given that the resolution effects are at a few percent level at most, the combined use of peak flux density and the adopted $\Omega^{2}$ yields lower limits to the brightness temperature figures.

The next section discusses the EVN imaging results within the framework of other properties known for these galaxies.

\section{Individual galaxies}

\subsection{NGC 2911}

The dominant radio core in NGC 2911 shows pronounced variability (Jones et al. 1982; Wrobel \& Heeschen 1984; Condon et al. 1991; Slee et al. 1994). From arcminute- and arcsecond-resolution observations it appears that about $10 \mathrm{mJy}$ of low-surface brightness radio emission is present along the major axis of the galaxy (Wrobel \& Heeschen 1984; Condon et al. 1991). Weak mas structure at $\mathrm{PA} \approx$ $-30^{\circ}$ was claimed to have been detected in an early 1.7 GHz VLBI experiment (Jones et al. 1981), but was not confirmed with $5 \mathrm{GHz}$ VLBI observations (Jones et al. 1982; Schilizzi et al. 1983).

We do not detect mas-scale extended emission. Our results show an unresolved, high-brightness temperature $\left(T_{\mathrm{B}}>10^{8} \mathrm{~K}\right)$ core with a flux density of $\sim 20 \mathrm{mJy}$. Longbaseline PTI (Parkes-Tindinbilla interferometer) measurements in the late 1980 s by Slee et al. (1994) yielded a relatively flat radio spectrum for the core; $\alpha=0.21$ between 2.3 and $8.4 \mathrm{GHz}$. Interpolating the PTI flux densities to $5 \mathrm{GHz}$ yields a value of $43 \mathrm{mJy}$, which, compared to the 163 mJy measured in 1980 with the VLA (Wrobel \& Heeschen 1984) and to our 1997 result, confirms the presence of strong variability. The presence of a variable, unresolved, flat-spectrum, high-brightness temperature radio core constitutes compelling evidence for an AGN-type source in this LINER galaxy.

\subsection{NGC 3079}

Arcsecond-resolution images of this well-known active galaxy show large-scale radio structure. Emission along the galactic disk and two 15-kpc nonthermal lobes emanate from the nucleus along the minor axis (de Bruyn 1977; Seaquist et al. 1978; Duric et al. 1983; Hummel et al. 1983; Duric \& Seaquist 1988; Baan \& Irwin 1995). This morphology, which is also seen in the X-rays (Fabbiano et al. 1992; Dahlem et al. 1998; Pietsch et al. 1998) and in optical line emission (Ford et al. 1986) has been interpreted as an outflow from a compact central engine that interacts with the dense gas in the nuclear environment (see discussion in Filippenko \& Sargent 1992). The hypothesis that this outflow may be driven by accretion onto a supermassive black hole is reinforced by high-resolution VLA and global VLBI observations (e.g., Irwin \& Seaquist 1988; Trotter et al. 1998; Sawada-Satoh et al. 2000) that reveal several aligned sources thought to be features of a nuclear jet.

Our EVN observations detect a slightly resolved, $\sim 15$ mJy radio core, in addition to a weak $(\sim 2 \mathrm{mJy})$ extension 17 mas toward the Southeast. From its flux density and position relative to the extended emission, we identify the core with the peaked-spectrum component "B" observed in the radio maps of Irwin \& Seaquist (1988), Trotter et al. (1998), and Sawada-Satoh et al. (2000). From comparison with the above-mentioned data, the $5 \mathrm{GHz}$ flux density of component $\mathrm{B}$ has been constant, within the errors, from 1986 through 1997. On the other hand, the $8 \mathrm{GHz}$ values of Trotter et al. (1998) and Sawada-Satoh et al. (2000) suggest variability at shorter wavelengths. Based on the probable variability and the high brightness temperature measured in this paper, we identify component B with the core of NGC 3079. The weak extended emission that we observe Southeast of B, but which is not well constrained by our data, may be associated with components "A" and "C" as seen in the previous studies. Based on their spectra, the multi-component nature, and the position of the extended structure, Trotter et al. (1998) and Sawada-Satoh et al. (2000) argue that A and $\mathrm{C}$ can be identified with the fading and/or expanding components in a radio jet.

\subsection{NGC 3998}

Low-resolution radio observations reveal arcminute-scale emission in NGC 3998: a structure consisting of a core and 
double lobes $\left(\sim 4^{\prime}\right.$ or $\left.20 \mathrm{kpc}\right)$ was detected with an overall PA of $\sim 0^{\circ}$ to $-15^{\circ}$ (Hummel 1980; Wrobel \& Heeschen 1984; Wrobel 1991), slightly misaligned relative to the galaxy minor axis. The radio core is variable and has a flat spectrum (e.g., Hummel et al. 1984).

Our high-resolution EVN observations detect a strong ( $\sim 83 \mathrm{mJy})$, slightly resolved core, consistent with the VLBI measurement ( $86 \mathrm{mJy}$ ) obtained by Hummel et al. (1982). The core displays a weak northern extension, which we suspect is the innermost part of the postulated kpc-scale outflow (Hummel 1980). The presence of a compact, variable, flat-spectrum, high-brightness temperature radio core associated with a jetlike extension constitutes compelling evidence for an AGN-type source in this LINER. This evidence is further strengthened by the detection of an X-ray (Roberts \& Warwick 2000; Terashima et al. 2000; Pellegrini et al. 2000) and ultraviolet (Fabbiano et al. 1994) point source, by the presence of a broad $\mathrm{H} \alpha$ emission line (Ho et al. 1997c), and by the inferred presence of a $10^{8} M_{\odot}$ black hole (Dressel et al. 2000).

\subsection{NGC 6500}

Arcsecond-scale radio emission has been detected in this source by Unger et al. (1989), who find two-sided extended emission up to $5^{\prime \prime}$ from the nucleus at $P A=140^{\circ}$, roughly perpendicular to the galaxy major axis. Unlike the highly collimated jet emission seen in radio galaxies, this arcsecond-scale emission has a wide opening angle of $\sim 60^{\circ}$. The radio morphology has been interpreted as evidence for an outflow along the minor axis of the galaxy (Unger et al. 1989), similar to that seen in NGC 3079 (see Sect. 5.2). On the other hand, the central 1."3 extended emission seen in the $408 \mathrm{MHz}$ and $1.7 \mathrm{GHz}$ MERLIN maps (Unger et al. 1989) is roughly aligned with the major axis of the galaxy, at $P A=55^{\circ}$ and $70^{\circ}$, respectively, consistent with early VLBI experiments by Jones et al. (1981, 1982). More recently, the $5 \mathrm{GHz}$ VLBA observations of Falcke et al. (2000) show a core straddled by twosided emission (overall size 20 mas, $P A=39^{\circ}$ ), aligned to within $9^{\circ}$ of the extended optical emission-line gas detected by Gonzaléz Delgado \& Pérez (1996). The misalignment between the small and large scales may be due to projection effects, or, alternatively, the jet may be disrupted and redirected very near the core. NGC 6500 is also known to be variable in the radio (e.g., Hummel et al. 1984) and has a flat radio spectrum (e.g., Falcke et al. 2000).

Our high-resolution observations show a strong ( $\sim 84 \mathrm{mJy}$ ), marginally resolved core consistent with the VLBA (Falcke et al. 2000) and VLA (Nagar et al. 2000) measurements. We do not detect the extended emission reported by Falcke et al. (2000), most likely because of the high noise level in our EVN data. The presence of an unresolved, variable, flat-spectrum, high-brightness temperature radio core associated with jetlike emission again provide compelling evidence for an AGN-type source, especially when considered in conjunction with the X-ray (Barth et al. 1997) and ultraviolet (Barth et al. 1997, 1998; Maoz et al. 1998) detections.

\section{Discussion and summary}

The physical origin of LINERs has been a controversial topic since Heckman (1980) identified them as a major constituent of the galaxy population. In recent years, high-resolution, multiwavelength observations have contributed greatly to elucidating the nature of these enigmatic objects. As discussed recently by Barth (2001) and Ho (2001), there is now little doubt that "type 1" LINERs (those with detectable broad emission lines) are genuine low-luminosity AGNs.

An outstanding issue yet to be resolved is the AGN fraction among narrow-lined, "type 2" LINERs and socalled transition objects. The traditional optical diagnostic emission lines are largely degenerate with respect to a number of the ionization mechanisms that have been proposed (see Sect. 1). While the ultraviolet region can be advantageous compared to the optical, the detection rate in this band is low (Maoz et al. 1995; Barth et al. 1998), most likely due to a combination of dust extinction (Barth et al. 1998; Pogge et al. 2000) and the intrinsic weakness of LINERs in this spectral region (Ho 1999b, 2001). In the few cases where ultraviolet spectroscopy is available, the evidence for AGNs has been mixed (Maoz et al. 1998; Shields et al. 2001).

A method widely used to discriminate AGNs from starburst-dominated sources compares the relative strength of the far-infrared flux to the radio flux. For "normal" or star-forming galaxies, Condon \& Broderick (1988) find that the distribution of the $u$ parameter, defined as the logarithmic ratio of the flux densities at $60 \mu \mathrm{m}$ and $1.4 \mathrm{GHz}$, peaks at $u \approx 2.0$, with a tail toward lower values of $u$ (excess radio emission) due to galaxies containing prominent AGNs. AGNs also generally have "warmer" far-infrared spectra compared to star-forming galaxies (e.g., de Grijp et al. 1985), which are characterized by $\alpha_{\mathrm{IR}} \approx 2.3-3.0$ between 25 and $60 \mu \mathrm{m}$ (Condon \& Broderick 1988; Condon et al. 1991). We have calculated $u$ and $\alpha_{\mathrm{IR}}$ for our sample (Table 3 ) using the far-infrared measurements tabulated in Ho et al. (1997a) and the integrated $1.4 \mathrm{GHz}$ flux densities from the NVSS (see Table 1). Indeed, all three galaxies with a dominant nuclear radio component (NGC 2911, NGC 3998, and NGC 6500) do have a low value of the $u$ parameter $(<1)$ and a relatively flat infrared spectrum $\left(\alpha_{\mathrm{IR}} \lesssim 2\right)$. (NGC 3079 is more ambiguous, but this is not surprising in view of the circumstantial evidence for strong star formation suggested by its optical morphology.) However, the application of these infrared diagnostics depends on the relative strength of the nuclear radio emission.

The X-ray band, especially for energies above $2 \mathrm{keV}$, provides a more promising tool to probe the central source in LINERs. However, until the recent advent of Chandra 
(Ho et al. 2001), previous hard X-ray observations of these sources (e.g., Ptak et al. 1999; Terashima et al. 2000) relied on the coarse beam presented by $A S C A$. Given the complexity of the X-ray structure in the central regions of nearby galaxies (e.g., Ho et al. 2001), the low-resolution $A S C A$ data also can yield ambiguous results for the less prominent nuclei that usually characterize type 2 LINERs.

This paper illustrates that radio VLBI observations can be added to the arsenal of tools to tackle the LINER problem, and, moreover, that the radio data alone can give meaningful physical constraints. We have obtained mas-resolution $5 \mathrm{GHz}$ observations of a small, but representative, sample of LINERs. The radio maps enable us to pinpoint highly compact central cores with sizes $\lesssim 1.5$ light year, which in turn place stringent lower limits on brightness temperatures $\left(\gtrsim 10^{8} \mathrm{~K}\right)$ that definitively rule out a thermal origin for the radio emission. The nonthermal, AGN-like nature of the radio sources is further suggested by other radio characteristics found in previous observations. These include the detection of source variability, flat or inverted spectra ${ }^{1}$, and in three out of the four cases, morphological evidence for jetlike features or outflows. All of the above are hallmark features of "classical" AGNs, the distinction being that the luminosities of our sources are several orders of magnitude lower than those observed in traditional radio galaxies.

Acknowledgements. M. E. F. acknowledges support from the Fundação para a Ciência e Tecnologia, Ministério da Ciência e Tecnologia, Portugal through the grant PRAXIS XXI/BD/15830/98. M. E. F. would like to thank Denise Gabuzda and Lorant Sjouwerman at JIVE (Joint Institute for VLBI) for their help on the data reduction. We also thank the staff of the EVN observatories and the MkIII VLBI Correlator at MPIfR, Bonn. The work of L. C. H. is partly funded by NASA grants from the Space Telescope Science Institute (operated by AURA, Inc., under NASA contract NAS5-26555). This research has made extensive use of NED (NASA/IPAC Extragalactic Database), which is operated by the Jet Propulsion Laboratory, California Institute of Tecnology, under contract with NASA.

\section{References}

Alonso-Herrero, A., Rieke, M. J., Rieke, G. H., \& Shields, J. C. 2000, ApJ, 530, 688

Baan, W. A., \& Irwin, J. A. 1995, ApJ, 446, 602

Barth, A. J. 2001, in Issues in Unification of AGNs, ed. R. Maiolino, A. Marconi, \& N. Nagar (San Francisco: ASP), in press

Barth, A. J., Filippenko, A. V., \& Moran, E. C. 1999, ApJ, 525,673

Barth, A. J., Ho, L. C., Filippenko, A. V., \& Sargent, W. L. W. 1998, ApJ, 496, 133

1 The spectral indices summarized in Table 2 derive from non-simultaneous observations, and therefore should be interpreted with some caution in light of the variable nature of the sources. Ulvestad \& Ho (2001) found flat-spectrum cores in three LINERs that were observed simultaneously at four frequencies with the VLBA.
Barth, A. J., Reichert, G. A., Ho, L. C., et al. 1997, AJ, 114, 2313

Barth, A. J., \& Shields, J. C. 2000, PASP, 112, 753

Becker, R. H., White, R. L., \& Edwards, A. L. 1991, ApJS, 75, 1

Becker, R. H., White, R. L., \& Helfand, D. J. 1995, ApJ, 450, 559

Condon, J. J., \& Broderick, J. J. 1988, AJ, 96, 30

Condon, J. J., Cotton, W. D., Greisen, E. W., et al. 1998, AJ, 115,1693

Condon, J. J., Frayer, D. T., \& Broderick, J. J. 1991, AJ, 101, 362

Dahlem, M., Weaver, K. A., \& Heckman, T. M. 1998, ApJS, 118,401

de Bruyn, A. G. 1977, A\&A, 58, 221

de Grijp, M. H. K., Miley, G. K., Lub, J., \& de Jong, T. 1985, Nature, 314, 240

Dopita, M. A., \& Sutherland, R. S. 1995, ApJ, 455, 468

Dressel, L. L., Ford, H. C., Kriss, G. A., \& Tsvetanov, Z. I. 2000, BAAS, 196, 21.04

Duric, N., \& Seaquist, E. R. 1988, ApJ, 326, 574

Duric, N., Seaquist, E. R., Crane, P. C., Bignell, R. C., \& Davis, L. E. 1983, ApJ, 273, 11

Fabbiano, G., Fassnacht, C., \& Trinchieri, G. 1994, ApJ, 434, 67

Fabbiano, G., Kim, D.-W., \& Trinchieri, G. 1992, ApJS, 80, 531

Falcke, H., Nagar, N. M., Wilson, A. S., \& Ulvestad, J. S. 2000, ApJ, 542, 197

Filippenko, A. V., \& Sargent, W. L. W. 1985, ApJS, 57, 503

Filippenko, A. V., \& Sargent, W. L. W. 1992, AJ, 103, 28

Filippenko, A. V., \& Terlevich, R. 1992, ApJ, 397, L79

Ford, H. C., Dahari, O., Jacoby, G. H., Crane, P. C., \& Ciardullo, R. 1986, ApJ, 311, L7

Fosbury, R. A. E., Mebold, U., Goss, W. M., \& Dopita, M. A. 1978, MNRAS, 183, 549

González Delgado, R. M., \& Pérez, E. 1996, MNRAS, 281, 1105

Halderson, E. L., Moran, E. C., Filippenko, A. V., \& Ho, L. C. 2001, AJ, 122, 637

Heckman, T. M. 1980, A\&A, 87, 152

Ho, L. C. 1999a, ApJ, 510, 631

Ho, L. C. 1999b, ApJ, 516, 672

Ho, L. C. 2001, in AGN Surveys, ed. R. F. Green, E. Ye. Khachikian, \& D. B. Sanders, IAU Colloq., 184 (San Francisco: ASP), 12

Ho, L. C., Feigelson, E. D., Townsley, L. K., et al. 2001, ApJ, 549, L51

Ho, L. C., Filippenko, A. V., \& Sargent, W. L. W. 1995, ApJS, 98, 477

Ho, L. C., Filippenko, A. V., \& Sargent, W. L. W. 1997a, ApJS, 112,315

Ho, L. C., Filippenko, A. V., \& Sargent, W. L. W. 1997b, ApJ, 487,568

Ho, L. C., Filippenko, A. V., Sargent, W. L. W., \& Peng, C. Y. 1997c, ApJS, 112, 391

Hummel, E. 1980, A\&AS, 41, 151

Hummel, E., Fanti, C., Parma, P., \& Schilizzi, R. T. 1982, A\&A, 114, 400

Hummel, E., van der Hulst, J. M., \& Dickey, J. M. 1984, A\&A, 134, 207

Hummel, E., van Gorkom, J. H., \& Kotanyi, C. G. 1983, ApJ, 267, L5

Irwin, J. A., \& Seaquist, E. R. 1988, ApJ, 335, 658 
Jones, D. L., Sramek, R. A., \& Terzian, Y. 1982, ApJ, 261, 422

Jones, D. L., Terzian, Y., \& Sramek, R. A. 1981, ApJ, 246, 28

Maoz, D., Filippenko, A. V., Ho, L. C., et al. 1995, ApJ, 440, 91

Maoz, D., Koratkar, A. P., Shields, J. C., et al. 1998, AJ, 116, 55

Nagar, N. M., Falcke, H., Wilson, A. S., \& Ho, L. C. 2000, ApJ, 542,186

Pellegrini, S., Cappi, M., Bassani, L., Della Ceca, R., \& Palumbo, G. G. C. 2000, A\&A, 360, 878

Pietsch, W., Trinchieri, G., \& Volger, A. 1998, A\&A, 340, 351

Pogge, R. W., Maoz, D., Ho, L. C., \& Eracleous, M. 2000, ApJ, 532,323

Ptak, A., Serlemitsos, P., Yaqoob, T., \& Mushotzky, R. 1999, ApJS, 120, 179

Roberts, T. P., \& Warwick, R. S. 2000, MNRAS, 315, 98

Sadler, E. M., Jenkins, C. R., \& Kotanyi, C. G. 1989, MNRAS, 240,591

Sandage, A., \& Bedke, J. 1994, The Carnegie Atlas of Galaxies (Washington, DC: Carnegie Inst. of Washington)

Sawada-Satoh, S., Inoue, M., Shibata,et al. 2000, PASJ, 52, 421

Schilizzi, R. T., Fanti, C., Fanti, R., \& Parma, P. 1983, A\&A, 126,412

Seaquist, E. R., Davis, L., \& Bignell, R. C. 1978, A\&A, 63, 199

Shields, J. C. 1992, ApJ, 399, L27
Shields, J. C., Sabra, B. M., Ho, L. C., Barth, A. J., \& Filippenko, A. V. 2001, in Mass Outflow in Active Galactic Nuclei: New Perspectives, ed. D. M. Crenshaw, S. B. Kraemer, \& I. M. George (San Francisco: ASP), in press

Slee, O. B., Sadler, E. M., Reynolds, J. E., \& Ekers, R. D. 1994, MNRAS, 269, 928

Terashima, Y., Ho, L. C., \& Ptak, A. F. 2000, ApJ, 539, 161

Terlevich, R., \& Melnick, J. 1985, MNRAS, 213, 841

Terlevich, R., Tenorio-Tagle, G., Franco, J., \& Melnick, J. 1992, MNRAS, 255, 71

Trotter, A. S., Greenhill, L. J., Moran, J. M., et al. 1998, ApJ, 495,740

Tully, R. B. 1988, Nearby Galaxies Catalog (Cambridge: Cambridge Univ. Press)

Ulvestad, J. S., \& Ho, L. C. 2001, ApJ, 562, 133

Unger, S. W., Pedlar, A., \& Hummel, E. 1989, A\&A, 208, 14

van Breugel, W. J. M., Schilizzi, R. T., Hummel, E., \& Kapahi, V. K. 1981, A\&A, 96, 310

van Moorsel, G., Kemball, A., \& Greisen, E. 1996, in Astronomical Data Anlaysis Software and Systems V, ed. G. H., Jacoby, \& J. Barnes (San Francisco: ASP), 37

White, R. L., \& Becker, R. H. 1992, ApJS, 79, 331

Wrobel, J. M. 1991, AJ, 101, 127

Wrobel, J. M., \& Heeschen, D. S. 1984, ApJ, 287, 41

Wrobel, J. M., \& Heeschen, D. S. 1991, AJ, 101, 148 